\title{
Temperature-Dependence of Exciton Radiative Recombination in (Al,Ga)N/GaN Quantum Wells Grown on a-Plane GaN Substrates
}

\author{
Pierre Corfdir ${ }^{1,2 *}$, Amélie Dussaigne ${ }^{1 \dagger}$, Henryk Teisseyre ${ }^{3,4}$, Tadeusz Suski ${ }^{3}$, Izabella Grzegory ${ }^{3}$, \\ Pierre Lefebvre ${ }^{5}$, Etienne Giraud ${ }^{1}$, Mehran Shahmohammadi ${ }^{1}$, Richard T. Phillips ${ }^{2}$, \\ Jean-Daniel Ganiere ${ }^{1}$, Nicolas Grandjean ${ }^{1}$, and Benoît Deveaud ${ }^{1}$
}

\author{
${ }^{1}$ Institute of Condensed Matter Physics, Ecole Polytechnique Fédérale de Lausanne, 1015 Lausanne, Switzerland \\ ${ }^{2}$ Cavendish Laboratory, University of Cambridge, CB3 OHE Cambridge, United Kingdom \\ ${ }^{3}$ Institute of High Pressure Physics, Polish Academy of Sciences, 01-142 Warsaw, Poland \\ ${ }^{4}$ Institute of Physics, Polish Academy of Sciences, 02-668 Warsaw, Poland \\ ${ }^{5}$ CNRS, Laboratoire Charles Coulomb, UMR5221, 34095 Montpellier, France \\ E-mail: pmc53@cam.ac.uk
}

Received October 12, 2012; accepted November 22, 2012; published online May 20, 2013

This article presents the dynamics of excitons in a-plane (Al,Ga)N/GaN single quantum wells of various thicknesses grown on bulk GaN substrates. For all quantum well samples, recombination is observed to be predominantly radiative in the low-temperature range. At higher temperatures, the escape of charge carriers from the quantum well to the $(\mathrm{Al}, \mathrm{Ga}) \mathrm{N}$ barriers is accompanied by a reduction in internal quantum efficiency. Based on the temperature-dependence of time-resolved photoluminescence experiments, we also show how the local disorder affects the exciton radiative lifetime at low temperature and the exciton non-radiative lifetime at high temperature.

(C) 2013 The Japan Society of Applied Physics

\section{Introduction}

One of the most important remaining bottlenecks regarding the use of III-nitride based light emitting diodes for high power applications is the reduction in efficiency at high injection current, the so-called "efficiency droop".1) Although the origin of the efficiency droop is still the subject of controversy, ${ }^{1-4)}$ many studies suggest that it could originate from efficient Auger recombination of charge carriers confined in the quantum well (QW) plane. ${ }^{2)}$ One natural way to decrease the efficiency of Auger recombination is to decrease the charge carrier density in the QW, which can be achieved by increasing the thickness of the QW. However, in GaN-based heterostructures grown along the [0001] axis, the discontinuity of the polarization field at the interfaces of the QW leads to a built-in electric field that spatially separates the electron and hole wave functions ${ }^{5)}$ and causes therefore a dramatic increase in radiative decay time for QW thicknesses larger than about $3 \mathrm{~nm}$. Non-polar planes allow for the growth of thick QWs with an optimal overlap between electron and hole wave functions, ${ }^{6)}$ but the use of lattice-mismatched substrates induces high densities of stacking faults and dislocations. Then, even at low temperature, the decay of excitons in non-polar QWs is dominated by their capture on extended defects. ${ }^{7,8)} \mathrm{We}$ have recently shown that depositing non-polar (Al,Ga)N/GaN QWs directly on the $a$-facet of GaN crystals allows for a significant increase of the radiative efficiency at $300 \mathrm{~K} .{ }^{9,10}$ ) Based on room-temperature cathodoluminescence experiments, we deduced a dislocation density of $2 \times 10^{5} \mathrm{~cm}^{-2}$ in the QW and an exciton diffusion length of $100 \mathrm{~nm}$, demonstrating that dislocations do not affect QW excitons at room temperature. ${ }^{9)}$ Then, from time-resolved photoluminescence (PL) measurements, we showed that the radiative efficiency at high temperature of these non-polar QW samples was related to the non-radiative recombination in

${ }^{\dagger}$ Present address: CEA-LETI, Minatec Campus, 17 rue des Martyrs, 39054 Grenoble, France. the $(\mathrm{Al}, \mathrm{Ga}) \mathrm{N}$ barriers of charge carriers thermally emitted from the QW plane. ${ }^{10)}$

In the present paper, we describe the dynamics of excitons in non-polar $(\mathrm{Al}, \mathrm{Ga}) \mathrm{N} / \mathrm{GaN}$ single $\mathrm{QW}$ samples deposited on $a$-plane $\mathrm{GaN}$ crystals. For all the investigated QW samples, the mechanism limiting the radiative efficiency at $300 \mathrm{~K}$ is related to the thermal escape of carriers from the $\mathrm{QW}$ to the $(\mathrm{Al}, \mathrm{Ga}) \mathrm{N}$ barriers. We detail in particular how disorder affects the dynamics of excitons confined in the QW plane. At low temperature, we observe spatial variations in the exciton radiative decay time, which we ascribe to fluctuations in localization centre density along the QW plane. At room temperature, we observe that the larger the density of localization centres in the QW, the shorter the QW PL decay time. This leads us to the conclusion that there is a spatial correlation between the density of localization centres in the QW and the density of non-radiative point defects in the $(\mathrm{Al}, \mathrm{Ga}) \mathrm{N}$ barriers.

\section{Experiments}

We study by non-resonant time-resolved PL experiments $\mathrm{Al}_{0.06} \mathrm{Ga}_{0.94} \mathrm{~N} / \mathrm{GaN}$ single QWs deposited by $\mathrm{NH}_{3}$ molecular beam epitaxy on the $a$-facet of GaN substrates produced by the high-pressure solution method. The investigated samples have QW widths of 2, 4, and $7 \mathrm{~nm}$. As reported in Ref. 9, the resulting QW structures exhibits a threading dislocation density of the order of $2 \times 10^{5} \mathrm{~cm}^{-2}$. For time-resolved PL experiments, the QW samples were excited with the third harmonic $(\lambda=280 \mathrm{~nm})$ of a Ti: $\mathrm{Al}_{2} \mathrm{O}_{3}$ laser with 2 ps pulse width and $80.7 \mathrm{MHz}$ repetition rate. We estimate the photogenerated carrier density to $5 \times 10^{10} \mathrm{~cm}^{-2}$. The emission from the QW samples was analysed with a monochromator (focal length of $36 \mathrm{~cm}$ and grating of $1200 \mathrm{~g} / \mathrm{mm}$ ) followed by a streak camera using a photon counting mode. Continuous-wave (cw) PL experiments have been carried out using a HeCd laser $(\lambda=325 \mathrm{~nm})$. The QW emission was analysed with a monochromator (focal length of $64 \mathrm{~cm}$ and 1200 grooves per $\mathrm{mm}$ grating) followed by detection on a Peltier-cooled CCD. 


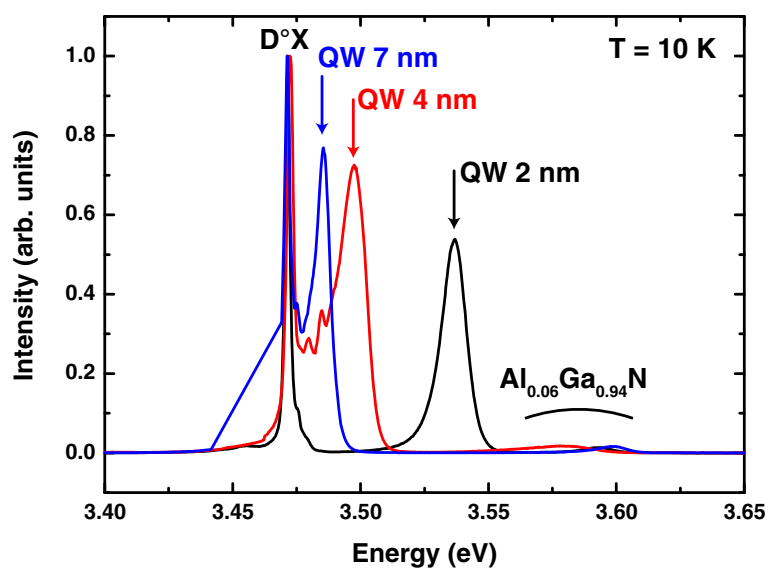

Fig. 1. (Color online) Time-integrated PL spectra taken at $10 \mathrm{~K}$ on 2-, 4-, and 7-nm-thick $\mathrm{Al}_{0.06} \mathrm{Ga}_{0.94} \mathrm{~N} / \mathrm{GaN}$ single QWs (black, red, and blue lines, respectively).

\section{Results and Discussion}

\subsection{Impact of disorder on the low-temperature exciton dynamics}

We display in Fig. 1 the cw-PL spectra at $10 \mathrm{~K}$ for the three QW samples. When increasing the QW thickness from 2 to $7 \mathrm{~nm}$, the QW emission energy decreases from 3.540 to $3.483 \mathrm{eV}$. From the dependence of the QW emission energy on the QW thickness, we confirm the absence of built-in electric fields in our samples. ${ }^{7)}$ In parallel, the emission linewidth goes from 13 to $8 \mathrm{meV}$, due to the reduced effect of interface roughness and barrier alloy disorder on the quantized energies for the thickest QWs. ${ }^{711)}$ We attribute the weak emission band observed in the $3.57-3.60 \mathrm{eV}$ range to the recombination of excitons in the $\mathrm{Al}_{0.06} \mathrm{Ga}_{0.94} \mathrm{~N}$ barriers. Concerning the narrow emission line centred at $3.471 \mathrm{eV}$, it corresponds to the recombination of donor bound excitons $\left(\mathrm{D}^{0} \mathrm{X}\right)$ in the GaN substrate. $\left.{ }^{9}{ }^{12}\right) \mathrm{We}$ also note the absence of any emission in the $3.42 \mathrm{eV}$ range, indicating the absence of basal plane stacking faults in our samples. ${ }^{13,14)}$ When moving the excitation spot across the samples, there is no noticeable change in QW emission energy and emission linewidth. This means that both the barrier Al-content and the QW thickness remain nearly constant across the sample. We present in Fig. 2 the PL decays $\tau_{\mathrm{PL}}$ measured on the 4-nm-thick QW sample with respect to temperature, for three different excitation spots. At $10 \mathrm{~K}$, excitons are mostly localized along the $\mathrm{QW}$ plane and $\tau_{\mathrm{PL}}$ is given by the radiative decay time of localized excitons $\tau_{\text {loc }}$. Scanning the excitation spot across the sample, the QW PL decay time at $10 \mathrm{~K}$ fluctuates between 105 and $130 \mathrm{ps}$. The decay time of localized excitons is inversely proportional to the coherence volume of the exciton, ${ }^{15)}$ which in the present case is given by the volume of the localization centre. ${ }^{16)}$ Consequently, as the QW thickness is constant across the sample, we attribute the variation in $\tau_{\text {loc }}$ to local change in the spatial extension of exciton localization centres.

At higher temperatures, excitons are delocalized along the QW plane and $\tau_{\mathrm{PL}}$ now corresponds to the decay of a thermalized distribution of localized and free QW excitons. ${ }^{17)}$ With $N_{\text {loc }}$ and $N_{\text {fr }}$ the density of localized and free QW excitons, respectively, one gets, in the simplest possible picture:

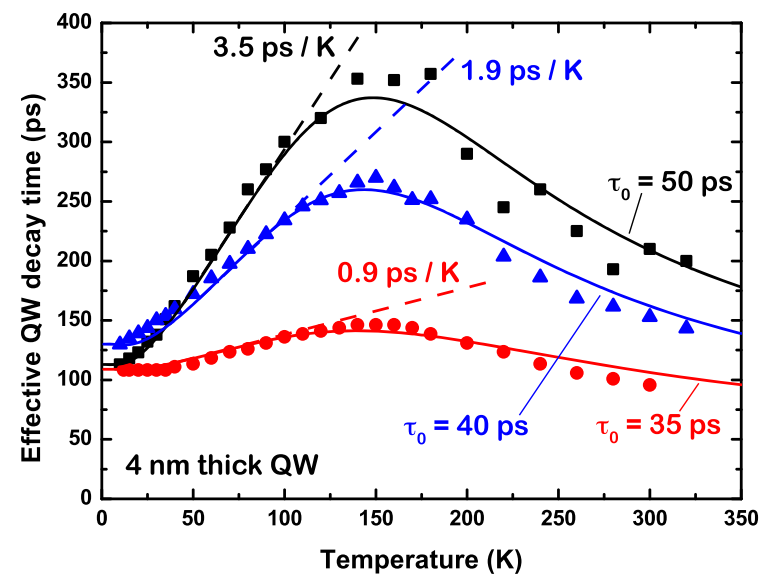

Fig. 2. (Color online) Effective QW PL decay time as a function of temperature for a 4-nm-thick $\mathrm{Al}_{0.06} \mathrm{Ga}_{0.94} \mathrm{~N} / \mathrm{GaN}$ single $\mathrm{QW}$, for three different excitation spots (symbols). The result of the fitting procedure using Eq. (1) is shown by solid lines. Dashed lines show the linear increase of exciton radiative lifetime with respect to temperature.

$$
\tau_{\mathrm{PL}}=\frac{N_{\mathrm{loc}}+N_{\mathrm{fr}}}{N_{\mathrm{loc}} / \tau_{\mathrm{loc}}+N_{\mathrm{fr}} / \tau_{\mathrm{fr}}}
$$

The free exciton decay time $\tau_{\mathrm{fr}}$ is given by $\tau_{\mathrm{fr}}^{-1}=\tau_{\mathrm{r}}^{-1}+\tau_{\mathrm{nr}}^{-1}$, where $\tau_{\mathrm{r}}$ and $\tau_{\mathrm{nr}}$ are $\mathrm{QW}$ free exciton radiative and nonradiative lifetimes, respectively. The density of localized excitons $N_{\text {loc }}$ is a function of temperature $(T)$, of the localization centre density $N_{\mathrm{D}}$ and of the exciton localization energy $E_{\text {loc }}$. As shown in Refs. 9 and 17, it is possible to express the ratio between localized and free exciton densities as

$$
\frac{N_{\mathrm{loc}}}{N_{\mathrm{fr}}}=\frac{N_{\mathrm{D}} \pi \hbar^{2}}{2 M k T} \exp \left(\frac{E_{\mathrm{loc}}}{k T}\right),
$$

where $M$ is the exciton mass. To compute $E_{\text {loc }}$, we use envelope function calculations that include a variational modeling of excitonic effects. ${ }^{18)}$ We assume that exciton localization occurs on monolayer fluctuation of the QW thickness ${ }^{19)}$ and we get $E_{\text {loc }}=8,3$, and $1 \mathrm{meV}$ for the 2-, 4-, and 7-nm-thick QWs. The computed QW width dependence of $E_{\text {loc }}$ agrees qualitatively with the localization energies deduced from the temperature-dependent PL experiments reported in Ref. 10. In addition, since we do not observe any change in QW emission linewidth when scanning across a sample of given QW thickness, we assume that $E_{\text {loc }}$ is constant along the QW plane. Regarding $\tau_{\text {loc }}$, it only depends on the coherence volume of the localized exciton ${ }^{15,16)}$ and we take it to be constant with temperature. In contrast, $\tau_{\mathrm{r}}$ depends on $M$ and on the oscillator strength $f_{\text {osc }}$, and increases linearly with temperature, due to the population of states lying out of the light cone (i.e., exciton states that do not couple to light). ${ }^{20,21)}$ Consequently, in the absence of non-radiative recombination, exciton delocalization leads to an increase in $\tau_{\mathrm{PL}}$. Experimentally, between 10 and $150 \mathrm{~K}$, we observe that $\tau_{\mathrm{PL}}$ for the 4-nm-thick QW sample increases with temperature (Fig. 2), proving the dominance of radiative recombination over non-radiative processes in this temperature range. Consistent with this, we find that for temperature below $150 \mathrm{~K}$, the QW emission intensity remains nearly constant (Fig. 3). 


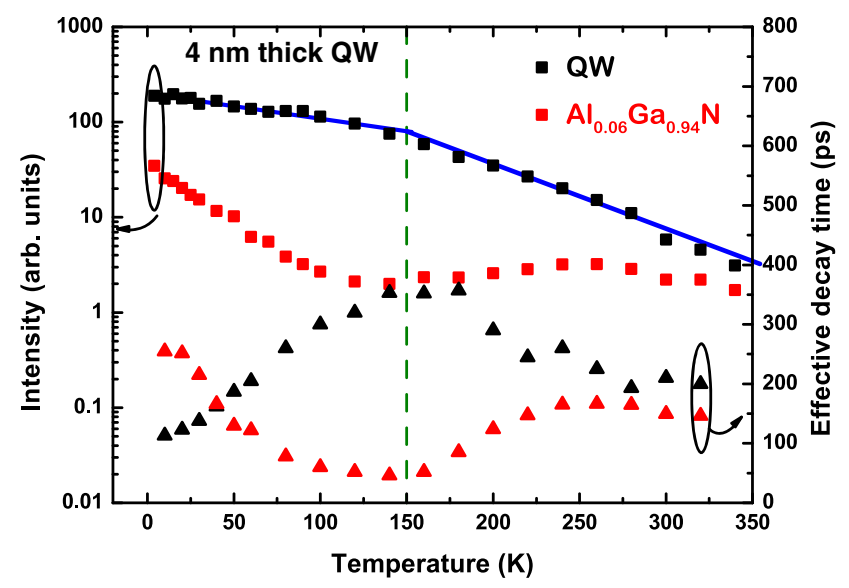

Fig. 3. (Color online) $\mathrm{QW}$ (black) and $\mathrm{Al}_{0.06} \mathrm{Ga}_{0.94} \mathrm{~N}$ barrier (red) emission intensity (squares) and effective decay time (triangles) as a function of temperature for a 4-nm-thick $\mathrm{Al}_{0.06} \mathrm{Ga}_{0.94} \mathrm{~N} / \mathrm{GaN}$ single $\mathrm{QW}$. Solid and dashed lines are guides to the eye.

It is to be noted that the gradient of $\tau_{\mathrm{PL}}$ is not the same over the whole sample. We observe that this gradient lies between 0.9 and $3.5 \mathrm{ps} / \mathrm{K}$ (Fig. 2). Using the formalism presented in Ref. 9, and assuming that $M$ and $f_{\text {osc }}$ do not depend on the local disorder amplitude, the increase of $\tau_{\mathrm{PL}}$ with temperature in the low-temperature range can be fitted with only a single free parameter: the density of localization centres $N_{\mathrm{D}}$. We deduce from the data displayed in Fig. 2 that $N_{\mathrm{D}}$ varies between $2 \times 10^{12}$ and $2 \times 10^{13} \mathrm{~cm}^{-2}$. We emphasize that we do not evidence any correlation between the coherence volume of localized exciton and the density of exciton localization centre. Note also that the localization centre densities computed here should be taken as indications of the relative local disorder amplitude rather than as absolute values characterizing the QW interface roughness at the local scale.

\subsection{Impact of disorder on the high-temperature exciton dynamics}

For temperatures higher than $150 \mathrm{~K}$, we observe for the 4nm-thick QW sample a decrease in $\tau_{\mathrm{PL}}$ and in QW emission intensity, indicating the activation of a non-radiative recombination process. It is worth noting that whatever the local density of localization centres, $\tau_{\mathrm{PL}}$ starts decreasing above the same temperature $T=150 \mathrm{~K}$ (Fig. 2). In parallel, we observe between 150 and $250 \mathrm{~K}$ the increase of both the (Al,Ga)N barriers PL intensity and lifetime (Fig. 3). This demonstrates that the non-radiative recombination of $\mathrm{QW}$ excitons is activated at the same temperature as the thermal escape of carriers from the $\mathrm{QW}$ to the $(\mathrm{Al}, \mathrm{Ga}) \mathrm{N}$ barriers (Fig. 3). As the decay of carriers in $\mathrm{Al}_{0.06} \mathrm{Ga}_{0.94} \mathrm{~N}$ is mainly non-radiative for temperatures higher than $100 \mathrm{~K},{ }^{10)}$ we attribute the decrease in $\tau_{\mathrm{PL}}$ to the non-radiative recombination in the $(\mathrm{Al}, \mathrm{Ga}) \mathrm{N}$ barriers of charge carriers that have thermally escaped the QW. To account for this non-radiative recombination channel, we consider that the non-radiative recombination decay time is given by

$$
\tau_{\mathrm{nr}}(T)=\tau_{0} \exp \left(\frac{E_{\mathrm{a}}}{k T}\right),
$$

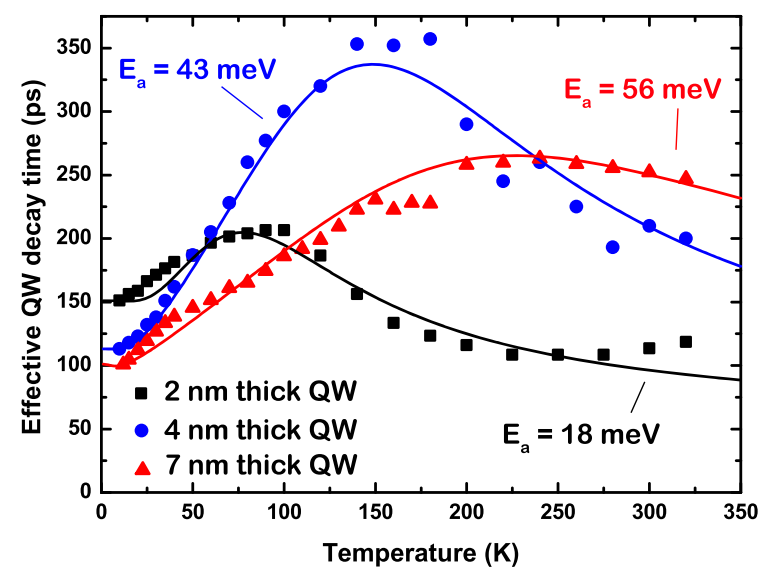

Fig. 4. (Color online) Effective QW PL decay time taken as a function of temperature for a 2 (black), 4 (red), and $7 \mathrm{~nm}$ (blue) thick $\mathrm{Al}_{0.06} \mathrm{Ga}_{0.94} \mathrm{~N} /$ GaN single QW samples. Solid lines show the result of the fitting procedure using Eq. (1).

where $E_{\mathrm{a}}$ is an activation energy that characterizes the depth of the QW and $\tau_{0}$ depends on details of the mechanisms of interaction between charge carriers and non-radiative recombination centres in the $(\mathrm{Al}, \mathrm{Ga}) \mathrm{N}$. Note, however, that in agreement with what reported in Ref. 22, the $E_{\mathrm{a}}$ obtained from temperature-dependent PL experiments cannot be directly compared to the electron and hole confinement energies. When aiming at describing the thermal escape of carriers from a QW, one needs to account for (i) the ionization of excitonic complexes into electron-hole pairs, ${ }^{10,23)}$ (ii) the difference in density of states between the $(\mathrm{Al}, \mathrm{Ga}) \mathrm{N}$ barriers and the GaN QW (three and two dimensional, respectively), ${ }^{10)}$ (iii) $\mathrm{B}$ and $\mathrm{C}$ excitons, as well as the excited states of the three exciton branches ${ }^{22)}$ and (iv) the disorder in both the QW plane and the $(\mathrm{Al}, \mathrm{Ga}) \mathrm{N}$ barriers. ${ }^{10,23)}$ As expected, we manage to fit the temperature dependence of $\tau_{\mathrm{PL}}$ for the different excitation spots keeping a constant $E_{\mathrm{a}}$, which we find equal to $43 \mathrm{meV}$ (Fig. 2). Regarding $\tau_{0}$, it ranges between 30 and 50 ps. As a matter of fact, we notice that $\tau_{0}$ and the local density in localization centres are correlated: the larger the local density of localization centres, the smaller $\tau_{0}$ and therefore the more efficient the non-radiative recombination in the $(\mathrm{Al}, \mathrm{Ga}) \mathrm{N}$ barriers.

Performing time-resolved PL experiments on the 2- and 7-nm-thick QW samples fully corroborates the results obtained on the 4-nm-thick QW sample (Fig. 4). First, we observe for all samples an increasing $\tau_{\mathrm{PL}}$ in the lowtemperature range, indicating purely radiative exciton recombination. We find that the local density of localization centres in the QW is the main parameter setting the temperature dependence of exciton radiative lifetime and consequently the slope of $\tau_{\mathrm{PL}}$ versus temperature. Second, and similarly to what is seen for the 4-nm-thick QW sample, we observe for the 2- and 7-nm-thick QW a decrease in $\tau_{\mathrm{PL}}$ in the high-temperature range. For a given barrier Alcontent, the thicker the $\mathrm{QW}$, the larger the temperature range of increasing $\tau_{\mathrm{PL}}$ (Fig. 4), as expected for non-radiative recombination processes related to the thermal escape of carriers from the QW to the barriers. ${ }^{10)}$ Using Eq. (1) to fit 
the temperature-dependence of $\tau_{\mathrm{PL}}$, we find $E_{\mathrm{a}}=18$ and $56 \mathrm{meV}$ for the 2- and 7-nm-thick QW samples, respectively, which verifies our earlier deductions. Coming to the values of $\tau_{0}$, we find them to be equal to $50 \pm 13$ and $66 \pm 15 \mathrm{ps}$, respectively, indicating that there is no relation between $\tau_{0}$ and the QW width, as one might have anticipated for nonradiative recombination occurring in the barriers.

\section{Conclusions}

In conclusion, we have performed time-resolved photoluminescence experiments on $a$-plane $(\mathrm{Al}, \mathrm{Ga}) \mathrm{N} / \mathrm{GaN}$ quantum wells grown on bulk GaN substrates for temperatures between 10 and $320 \mathrm{~K}$. From the increase with temperature of the exciton effective decay time in the quantum well together with the nearly constant integrated emission intensity, we show that exciton recombination is mainly radiative for temperatures below $150 \mathrm{~K}$. At higher temperatures, the decreases in effective decay time and emission intensity give evidences for the activation of non-radiative recombination, which we relate to the escape of carriers from the quantum well. We show that in the low-temperature range, variations in quantum well interface roughness lead to large fluctuations of exciton radiative decay time. We then show that the larger the density of localization centres in the quantum well, the more efficient the non-radiative recombination in the $(\mathrm{Al}, \mathrm{Ga}) \mathrm{N}$ barriers. This demonstrates a clear spatial correlation between disorder in the quantum well and in the $(\mathrm{Al}, \mathrm{Ga}) \mathrm{N}$ barriers.

\section{Acknowledgements}

We acknowledge financial support from the Swiss National Science Foundation through Project No. 129715 and from the Polish National Science Center (Project DEC-2011/ 03/B/ST3/02647). The work was partially supported by the European Union within European Regional Development Fund through Innovative Economy Grant No. POIG.01.01.02-00-008/08. P.C. also acknowledges financing from the European Union Seventh Framework Program under grant agreement No. 265073.
1) M. H. Kim, M. F. Schubert, Q. Dai, J. K. Kim, E. F. Schubert, J. Piprek, and Y. Park: Appl. Phys. Lett. 91 (2007) 183507.

2) E. Kioupakis, P. Rinke, K. T. Delaney, and C. G. Van de Walle: Appl. Phys. Lett. 98 (2011) 161107.

3) S. Hammersley, D. Watson-Parris, P. Dawson, M. J. Godfrey, T. J. Badcock, M. J. Kappers, C. McAleese, R. A. Oliver, and C. J. Humphreys: J. Appl. Phys. 111 (2012) 083512

4) B. Monemar and E. B. Sernelius: Appl. Phys. Lett. 91 (2007) 181103.

5) F. Bernardini and V. Fiorentini: Phys. Rev. B 57 (1998) R9427.

6) P. Waltereit, O. Brandt, A. Trampert, H. T. Grahn, J. Menniger, M. Ramsteiner, M. Reiche, and K. H. Ploog: Nature 406 (2000) 865.

7) A. Dussaigne, P. Corfdir, J. Levrat, T. Zhu, D. Martin, P. Lefebvre, J.-D Ganière, R. Butté, B. Deveaud-Plédran, N. Grandjean, Y. Arroyo, and P. Stadelmann: Semicond. Sci. Technol. 26 (2011) 025012.

8) T. J. Badcock, P. Dawson, M. J. Kappers, C. McAleese, J. L. Hollander, C. F. Johnston, D. V. Sridhara Rao, A. M. Sanchez, and C. J. Humphreys: Appl. Phys. Lett. 93 (2008) 101901.

9) P. Corfdir, J. Levrat, A. Dussaigne, P. Lefebvre, H. Teisseyre, I. Grzegory, T. Suski, J.-D. Ganière, N. Grandjean, and B. Deveaud-Plédran: Phys. Rev. B 83 (2011) 245326.

10) P. Corfdir, A. Dussaigne, H. Teisseyre, T. Suski, I. Grzegory, P. Lefebvre, E. Giraud, J.-D. Ganière, N. Grandjean, and B. Deveaud-Plédran: J. Appl. Phys. 111 (2012) 033517.

11) D. Bimberg, J. Christen, T. Fukunaga, H. Nakashima, D. E. Mars, and J. N. Miller: J. Vac. Sci. Technol. B 5 (1987) 1191.

12) K. Kornitzer, T. Ebner, K. Thonke, R. Sauer, C. Kirchner, V. Schwegler, M. Kamp, M. Leszczynski, I. Grzegory, and S. Porowski: Phys. Rev. B 60 (1999) 1471.

13) B. Bastek, F. Bertram, J. Christen, T. Wernicke, M. Weyers, and M. Kneissl: Appl. Phys. Lett. 92 (2008) 212111.

14) P. Corfdir, J. Ristic, P. Lefebvre, T. Zhu, D. Martin, A. Dussaigne, J.-D. Ganière, N. Grandjean, and B. Deveaud-Plédran: Appl. Phys. Lett. 94 (2009) 201115.

15) E. T. Rashba and G. E. Gurgenishvili: Fiz. Tverd. Tela (Leningrad) 4 (1962) 759 [in Russian].

16) J. Bellessa, V. Voliotis, R. Grousson, X. L. Wang, M. Ogura, and H. Matsuhata: Phys. Rev. B 58 (1998) 9933.

17) D. S. Citrin: Phys. Rev. B 47 (1993) 3832.

18) P. Corfdir and P. Lefebvre: J. Appl. Phys. 112 (2012) 053512.

19) C. Weisbuch, R. Dingle, A. C. Gossard, and W. Wiegmann: Solid State Commun. 38 (1981) 709.

20) L. C. Andreani, F. Tassone, and F. Bassani: Solid State Commun. 77 (1991) 641.

21) B. Deveaud, F. Clérot, N. Roy, K. Satzke, B. Sermage, and D. S. Katzer: Phys. Rev. Lett. 67 (1991) 2355.

22) J. S. Im, A. Moritz, F. Steuber, V. Härle, F. Scholtz, and A. Hangleiter: Appl. Phys. Lett. 70 (1997) 631.

23) P. Corfdir, J. Levrat, G. Rossbach, R. Butté, E. Feltin, J.-F. Carlin, G. Christmann, P. Lefebvre, J.-D. Ganière, N. Grandjean, and B. DeveaudPlédran: Phys. Rev. B 85 (2012) 245308. 